\title{
Bedside detection of awareness in the vegetative state: a cohort study
}

Damian Cruse, Srivas Chennu, Camille Chatelle, Tristan A Bekinschtein, Davinia Fernández-Espejo, John D Pickard, Steven Laureys, Adrian M Owen

\begin{abstract}
Summary
Background Patients diagnosed as vegetative have periods of wakefulness, but seem to be unaware of themselves or their environment. Although functional MRI (fMRI) studies have shown that some of these patients are consciously aware, issues of expense and accessibility preclude the use of fMRI assessment in most of these individuals. We aimed to assess bedside detection of awareness with an electroencephalography (EEG) technique in patients in the vegetative state.
\end{abstract}

Methods This study was undertaken at two European centres. We recruited patients with traumatic brain injury and non-traumatic brain injury who met the Coma Recovery Scale-Revised definition of vegtative state. We developed a novel EEG task involving motor imagery to detect command-following-a universally accepted clinical indicator of awareness-in the absence of overt behaviour. Patients completed the task in which they were required to imagine movements of their right-hand and toes to command. We analysed the command-specific EEG responses of each patient for robust evidence of appropriate, consistent, and statistically reliable markers of motor imagery, similar to those noted in healthy, conscious controls.

Findings We assessed 16 patients diagnosed in the vegetative state, and 12 healthy controls. Three (19\%) of 16 patients could repeatedly and reliably generate appropriate EEG responses to two distinct commands, despite being behaviourally entirely unresponsive (classification accuracy 61-78\%). We noted no significant relation between patients' clinical histories (age, time since injury, cause, and behavioural score) and their ability to follow commands When separated according to cause, two (20\%) of the five traumatic and one $(9 \%)$ of the 11 non-traumatic patients were able to successfully complete this task.

Interpretation Despite rigorous clinical assessment, many patients in the vegetative state are misdiagnosed. The EEG method that we developed is cheap, portable, widely available, and objective. It could allow the widespread use of this bedside technique for the rediagnosis of patients who behaviourally seem to be entirely vegetative, but who might have residual cognitive function and conscious awareness.

Funding Medical Research Council, James S McDonnell Foundation, Canada Excellence Research Chairs Program, European Commission, Fonds de la Recherche Scientifique, Mind Science Foundation, Belgian French-Speaking Community Concerted Research Action, University Hospital of Liège, University of Liège.

\section{Introduction}

Up to $43 \%$ of patients diagnosed as vegetative are reclassified as (at the least) minimally conscious when assessed by experienced teams. ${ }^{1-3}$ However, a further subset of conscious patients could exist who are undetected even after extensive clinical investigation in specialised centres. Findings from functional neuroimaging studies ${ }^{4.5}$ have called into question several of the core principles that underpin diagnosis of the vegetatative state; in particular, the extent to which clinicans can truly consider that a patient is unaware of themselves and their environment simply because they show no overt behavioural responses to external stimulation. For example, with functional MRI (fMRI), Owen and colleagues ${ }^{4}$ showed that a patient who seemed to be entirely vegetative was aware and able to modulate her blood oxygen-level dependent (BOLD) response to do various mental imagery tasks. With the same technique, Monti and colleagues ${ }^{5}$ showed that four (17\%) of 24 patients in the vegetative state were consciously aware and able to do these tasks reliably in the fMRI scanner. Moreover, one of the four patients could answer yes and no questions by modulating his fMRI response despite being unable to initiate any functional communication at the bedside. These findings confirm that a population of patients exists who meet all the behavioural criteria for the vegetative state, but nevertheless retain a level of covert awareness that cannot be detected by thorough behavioural assessment.

Use of fMRI in this patient group is very challenging; in addition to issues of cost and scanner availability, the physical stress incurred by patients when they are transferred to a suitably equipped fMRI facility is substantial. Movement artifacts often occur in imaging datasets from patients who are unable to remain still, and metal implants, including plates and pins, which are common in many traumatically injured populations, can completely rule out use of fMRI.

Electroencephalography (EEG) measures the activity of groups of cortical neurons from scalp electrodes, and
Published Online November 10, 2011 DOI:10.1016/S0140 6736(11)61224-5

See Online/Comment DOI:10.1016/S01406736(11)61591-2

Centre for Brain and Mind, University of Western Ontario, London, ON, Canada (D Cruse PhD, D Fernández-Espejo PhD Prof A M Owen PhD); Medical Research Council Cognition and Brain Sciences Unit (D Cruse, TA Bekinschtein PhD, Prof A M Owen) and Department of Clinical Neurosciences (S Chennu PhD) University of Cambridge, Cambridge, UK; Coma Science Group, Cyclotron Research Centre and Neurology Department, University and University Hospital of Liège, Liège, Belgium (C Chatelle MSC, Prof S Laureys MD); and Division of Academic Neurosurgery, Addenbrooke's Hopsital, Cambridge, UK (Prof J D Pickard MD)

Correspondence to: Dr Damian Cruse, Centre for Brain and Mind, University of Western Ontario, London, $\mathrm{ON}$, Canada N6A 5B7 dcruse@uwo.ca 
is much less expensive than MRI, both for initial cost and maintenance. EEG recordings are unaffected by metallic implants and, perhaps more importantly, can be used at the bedside. ${ }^{6}$ In the EEG record, imagined movements (motor imagery) are shown by reductions in power (event-related desynchronisations [ERD]) of the $\mu$ (about 7-13 Hz) or $\beta$ (about 13-30 Hz) frequency bands over the topographically appropriate areas of the motor cortex-eg, over the lateral premotor cortex for hand movements, and medial premotor cortex for toe movements. ${ }^{6}$ In some individuals, these ERDs might be accompanied by increases in power (event-related synchronisations [ERS]) over motor areas contralateral to, or surrounding, the ERD. ${ }^{8,9}$ With classification techniques, the form of motor imagery being done by a conscious individual can be accurately identified on the basis of these EEG responses alone. ${ }^{10}$ We investigated whether these general principles could be adapted to reliably detect covert conscious awareness in a convenience sample of patients who seemed to be entirely vegetative on the basis of repeated and thorough clinical assessment by specialist teams.

\section{Methods}

\section{Patients and controls}

This study was undertaken at two European centres (Addenbrooke's Hospital, Cambridge, UK, and University Hospital of Liège, Belgium), between July, 2010, and June, 2011. We recruited patients with traumatic brain injury and non-traumatic brain injury who met the coma recovery scale-revised (CRS-R $)^{11}$ definition of vegetative state. We recruited healthy control participants from the School of Social Sciences, University of Western Ontario, Canada.

We acquired informed assent from all patients' families and medical teams. For patients tested in Cambridge, ethics approval was provided by the National Research Ethics Service (National Health Service, UK); for those tested in Liège, approval was provided by the ethics committee of the University Hospital and Faculty of Medicine of the University of Liège. We obtained informed consent from all controls before the study. Ethics approval was provided by the Psychology Research Ethics Board.

\section{Procedures}

All patients were admitted for 4-5 days as part of a separate protocol and were assessed with the CRS-R on every day. This scale was developed to differentiate between patients in the vegetative state and those who are minimally conscious, and includes six subscales addressing auditory, visual, motor, oromotor, communication, and arousal functions.

We separated the EEG task into two blocks comprising right-hand imagery and toe imagery. All patients completed at least four (range four to eight) blocks of each type of movement, dependent on their level of agitation at the time of assessment. All controls completed six blocks. Block order was pseudorandomised so no more than two blocks of the same imagery type were completed consecutively. Each block began with the auditory presentation of the task instructions for that block. For the right-hand and toe blocks, respectively, the instructions were: "Every time you hear a beep, try to imagine that you are squeezing your right-hand into a fist and then relaxing it/wiggling all of the toes on both your feet, and then relaxing them. Concentrate on the way your muscles would feel if you were really performing this movement. Try to do this as soon as you hear each beep". After $5 \mathrm{~s}$, the instructions were followed by the binaural presentation of 15 tones $(600 \mathrm{~Hz}$ for $60 \mathrm{~ms})$ with an interstimulus interval of $4 \cdot 5-9.5 \mathrm{~s}$ (the stimulus computer was programmed to choose the interstimulus interval at random on each trial, with MATLAB). Each block concluded with an instruction to relax. All participants had a short break of 1-2 min before the start of the next block. All controls completed a task identical to the patients' motor imagery task, except that they were instructed by the experimenter to listen to the instruction and then mind-wander (ie, not to follow the commands) during the block. The stimulus computer was programmed to select the order of task completion at random for each healthy participant.

We recorded EEG responses from either a 129-channel electrode cap (Electrical Geodesics Inc, Oregon, USA; Cambridge, UK, and London, Onatario) or a 257-channel electrode cap (Liège) referenced to the vertex. To equalise the number of channels between patients, the 129 channels corresponding to those in the 129-channel electrode cap were selected from the 257-channel cap. This step ensured that the same number of EEG features were used for classification of motor imagery, and that accuracies were similar between centres. We filtered data offline between $1 \mathrm{~Hz}$ and $40 \mathrm{~Hz}$, segmented into epochs of $5.5 \mathrm{~s}$ (including $1.5 \mathrm{~s}$ before every tone), and baseline corrected in $500 \mathrm{~ms}$ before the tone. We identified bad channels by inspection (channel variance of about $>250$ ) and replaced them with interpolations of their neighbours (InvDist, EEGLAB ${ }^{12}$ ). We re-referenced all channels offline, including the online reference, to the average of their four geodesically nearest neighbours with a laplacian operator. This method of local average referencing produces focal patterns of ERD and ERS. ${ }^{13}$ We excluded trials containing large movement artifacts, to ensure the EEG recording consists of more purely brain activity and not muscle artifacts.

A median of 114 (range 60-202) trials contributed to each patient's single-trial analysis. We selected the 25 electrodes over the motor area (covering the area centrally from C3 to $\mathrm{C} 4$ [figure 1]) from the original 129-channel electrodes to contribute to the single-trial classification, because activity related to motor imagery is localised to this area of the scalp. A median of two (range zero to eight) channels from these 25 electrodes 
were interpolated before the analyses. The median number of trials contributing to the controls' analyses was 171 (range 154-180), with a median of one (zero to six) interpolated channel.

\section{Classification and statistical analyses}

For each participant, a linear support vector machine ${ }^{14}$ classifier was trained with the filtered and artifact-rejected data to classify trials into either right-hand or toe motor imagery. EEG data for the 25 electrodes selected across the motor cortex in every trial were downsampled to $100 \mathrm{~Hz}$. At each timepoint, we calculated log power values within the $\mu(7-13 \mathrm{~Hz})$, low- $\beta$ (13-19 Hz), middle- $\beta$ $(19-25 \mathrm{~Hz})$, and high- $\beta(25-30 \mathrm{~Hz})$ frequency ranges. All the band-power values in the period between $0.5 \mathrm{~s}$ and $3 \cdot 5 \mathrm{~s}$ after the tone (action period) were then concatenated by channel and used to construct a single feature vector for each trial. This technique allowed the classifier to be trained on discriminative spatiotemporal patterns in the EEG for the two types of motor imagery. We used blockwise cross-validation to identify the classifier's generalisation error for all the dataset. Specifically, the classifier was repeatedly trained and tested by excluding two blocks at a time (one right-hand and one toe block), training on the remaining blocks, and testing the generated support vector machine with the excluded blocks. During every repetition, we normalised the $Z$ scores of features in the training and test set with the mean and SD of the training set. This blockwise crossvalidation procedure, in addition to the pseudorandomised block order, ensured that task-irrelevant intrablock and interblock correlations in the EEG did not significantly account for the classification results.

To estimate overall accuracy for a patient or control, we used MATLAB's binofit function to concatenate and model as a binomial process all the binary single-trial classification outcomes from the blockwise cross-validation procedure. This procedure assumed that the individual classification outcomes were binomially distributed, and calculated the maximum likelihood estimate of the overall probability of correct classification. We then converted these estimates to percentage accuracy scores. Finally, we used a test of whether the $99 \%$ and $99.9 \%$ binomial CIs for the estimates included chance (50\%) to ascribe a significance level to each score. To confirm that significance could not occur because of overall changes in background EEG, which were not relevant to tasks and covaried with the pseudorandomised block order, we applied the described analyses to band-power features from a baseline period $500 \mathrm{~ms}$ wide and starting $500 \mathrm{~ms}$ before each tone. We deemed the classification accuracy in the action period after each tone to be significantly greater than the classification accuracy in this baseline period if it was outside the binomial CIs for the baseline accuracy. These comparisons ensured not only that classification accuracy was significant after each tone, but also that it was non-significant before the tone, and then increased

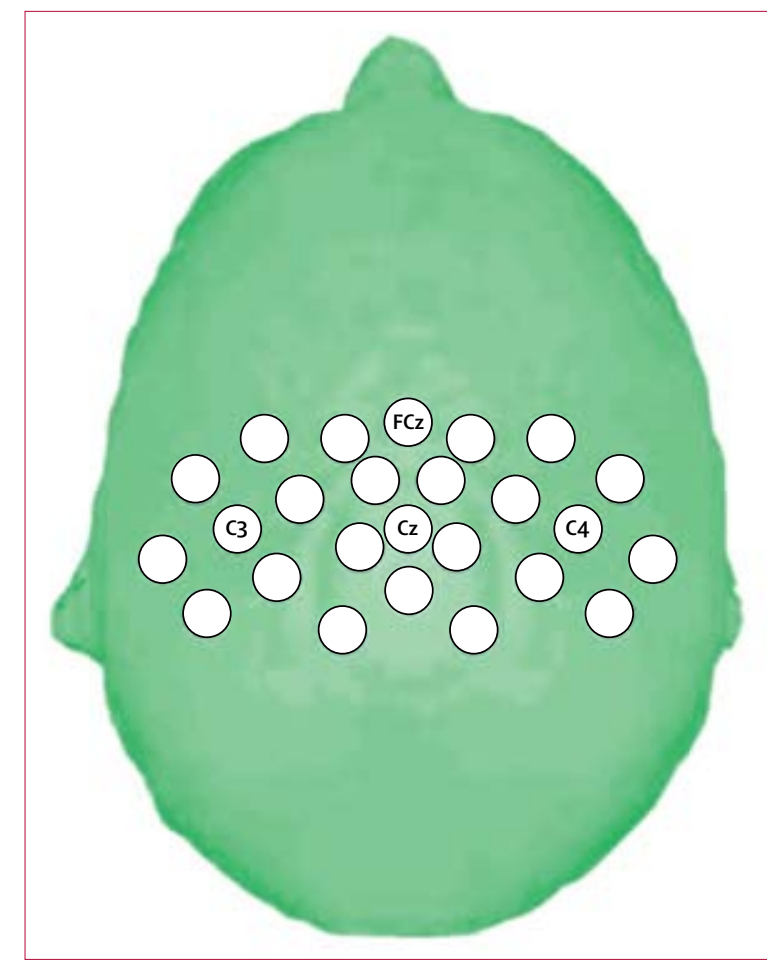

Figure 1: Scalp locations of the 25 electrodes contributing to the classification analyses

significantly after it. Therefore, the classification accuracy in the action period was generated by consistently timed motor imagery started after each tone.

We did all calculations in MATLAB with a combination of custom scripts, EEGLAB ${ }^{12}$ functions, and the $g$. BSanalyze software (version 3.10.00). We used SPSS software (version 19) for statistical analyses of the relation between aspects of patients' clinical history and their ability to follow command with this EEG task (linear and logistical regressions).

\section{Role of the funding source}

The sponsor of the study had no role in study design, data collection, data analysis, data interpretation, or writing of the report. The corresponding author had full access to all the data in the study and had final responsibility for the decision to submit for publication.

\section{Results}

We assessed 16 patients in the vegetative state (table). Patients with non-traumatic injuries were significantly older than were those with traumatic brain injuries $(p=0 \cdot 02)$. Median age of patients with traumatic brain injury was 29 years (range 14-45), whereas those with non-traumatic brain injury had a median age of 44 years (30-63). Controls had a median age of 25 years (range 21-31), were English speakers, and reported no neurological disorders. Time since injury $(\mathrm{p}=0 \cdot 13)$ and CRS-R score $(\mathrm{p}=0 \cdot 20)$ did not differ significantly between 


\begin{tabular}{|c|c|c|c|c|c|c|c|c|}
\hline & Sex & $\begin{array}{l}\text { Age at } \\
\text { assessment } \\
\text { (years) }\end{array}$ & $\begin{array}{l}\text { Interval } \\
\text { postictus } \\
\text { (months) }\end{array}$ & $\begin{array}{l}\text { Cause } \\
\text { (TBI/non-TBI) }\end{array}$ & CRS-R & $\begin{array}{l}\text { Number of tasks } \\
\text { contributing to } \\
\text { analyses }\end{array}$ & $\begin{array}{l}\text { EEG classification } \\
\text { accuracy }(\%)\end{array}$ & $\begin{array}{l}p \text { value for EEG } \\
\text { command following }\end{array}$ \\
\hline Patient 1 & Male & 35 & 9 & Anoxia & 7 & 202 & $61 \cdot 38 \%$ & $<0.01$ \\
\hline Patient 2 & Male & 63 & 39 & Anoxia & 5 & 113 & $61 \cdot 90 \%$ & NS \\
\hline Patient 3 & Male & 55 & 21 & Anoxia & 4 & 160 & $47 \cdot 50 \%$ & NS \\
\hline Patient 4 & Male & 35 & 32 & Anoxia & 6 & 69 & $43 \cdot 47 \%$ & NS \\
\hline Patient 5 & Male & 30 & 24 & Anoxia & 6 & 102 & $51.96 \%$ & NS \\
\hline Patient 6 & Female & 41 & 56 & Anoxia & 5 & 132 & $53 \cdot 78 \%$ & NS \\
\hline Patient 7 & Male & 63 & 32 & Anoxia & 7 & 76 & $56 \cdot 58 \%$ & NS \\
\hline Patient 8 & Female & 44 & 1 & Anoxia & 3 & 86 & $48.83 \%$ & NS \\
\hline Patient 9 & Male & 48 & 94 & Anoxia & 6 & 116 & $58.62 \%$ & NS \\
\hline Patient 10 & Female & 36 & 77 & Stroke & 3 & 114 & $39 \cdot 47 \%$ & NS \\
\hline Patient 11 & Male & 62 & 1 & Stroke & 6 & 142 & $48 \cdot 59 \%$ & NS \\
\hline Patient 12 & Male & 45 & 23 & Trauma & 6 & 146 & $71.23 \%$ & $<0.001$ \\
\hline Patient 13 & Male & 29 & 3 & Trauma & 6 & 96 & $78.13 \%$ & $<0.001$ \\
\hline Patient 14 & Male & 29 & 16 & Trauma & 6 & 150 & $40 \cdot 70 \%$ & NS \\
\hline Patient 15 & Male & 14 & 18 & Trauma & 6 & 60 & $41.66 \%$ & NS \\
\hline Patient 16 & Male & 21 & 7 & Trauma & 7 & 98 & $47 \cdot 95 \%$ & NS \\
\hline
\end{tabular}

the two injury groups (table). At no point during the 4-5 days when patients were admitted as part of a separate protocol did any patient show behaviour inconsistent with a diagnosis of vegetative state. Three $(19 \%)$ of 16 patients were able to follow the commands to a significantly detectable degree with the EEG assessment used (table). The classification accuracies for these three patients ranged from $61 \%$ to $78 \%$ (mean $70 \%$ ). None of the EEG recordings for the three patients were significant during the baseline period $(500 \mathrm{~ms}$ before each tone; mean 56\%; all p $>0.05$ ). For all three patients, the classification accuracy in the time after each tone was significantly greater $($ all $\mathrm{p}<0 \cdot 01)$ than that achieved in the baseline period (data not shown).

When separated according to cause, two (40\%) of the five patients (all $\mathrm{p}<0 \cdot 001)$ and one $(9 \%)$ of the 11 patients with non-traumatic brain injury $(\mathrm{p}<0 \cdot 01)$ returned positive EEG outcomes. Classification accuracies did not differ significantly between the traumatic and non-traumatic subgroups (mean $48 \%$ vs 52\%; $\mathrm{p}=0 \cdot 96$ ), nor in the proportions of patients significantly following commands (Fisher's exact test; $\mathrm{p}=0 \cdot 214)$. Nine $(75 \%)$ of the 12 healthy controls produced EEG data that could be classified significantly above chance (all $\mathrm{p}<0 \cdot 01)$. The accuracies for these nine participants ranged from $60 \%$ to $91 \%$ (mean $68 \%$ ), with the three remaining controls producing EEG that could be classified only between $44 \%$ and $53 \%$. During completion of the control task-ie, listening to the same imagery task as for patients, but not following commandsno healthy control participant returned EEG responses that could be classified significant according to the commands (mean 51\%, range 45-58\%; all p>0 .05).
A stepwise multiple linear regression analysis, including age at time of injury (months); time since injury (months), CRS-R score, and cause (traumatic or non-traumatic brain injury), did not significantly predict classification accuracy. A binary logistical regression analysis with the same factors as the stepwise analysis failed to predict positive EEG outcome (significant or otherwise). These results show that prediction of a patient's ability to follow commands in this EEG task is not possible on the basis of these aspects of their clinical history.

\section{Discussion}

Three study patients who seemed to be entirely vegetative on the basis of repeated specialist behavioural assessment were found to be aware and capable of substantially and consistently modulating their EEG responses to command. Our findings show that this EEG method can identify covert awareness in patients diagnosed in the vegetative state with a similar degree of accuracy to other methods of detection; it is a considerably cheaper and more portable bedside technique. The clinical history of study individuals had no effect on their ability to follow command.

Standard clinical assessments of command following based on behavioural observation are fundamentally subjective. Results of fMRI studies suggest that up to $17 \%(4 / 23)$ of patients diagnosed as in the vegetative state after behavioural assessment can follow commands when such commands need a change in bloodoxygenation level dependent reponse, rather than overt motoric behaviour. ${ }^{4,5}$ 
To fully appreciate our findings, the criteria that had to be met before a significant EEG result is produced should be considered. First, patients had to modulate the appropriate frequency bands of the EEG signal that are associated with motor imagery, over the same regions of the head where this activity is known to occur in aware individuals (figure 2). Second, for each type of imagery to be accurately classified, this modulation had to be consistent across tasks of the same imagery typeie, with a consistent time course and frequency contentbut had to also differ consistently between the two types of imagery (right-hand and toe). Finally, the classification of the patient's EEG data had to be significant in a binomial test.

Could appropriate patterns of activity be elicited in these patients in the absence of awareness? Could they somehow indicate an automatic, rather than a conscious and overt, response to aspects of the task instructions, such as to the words right-hand and toes? This scenario is extremely unlikely and we know of no data to support such a conclusion with a task like the one used in our study. The task instructions were delivered once at the beginning of each block of 15 cues (short tones) that signalled the time to begin each imagery trial. Any automatic response to the previously presented verbal instruction would then have to abate and recur in synchrony with these cues, which carried no information about the task to be undertaken. Three-quarters of the control participants had significant EEG outcomes when completing this motor imagery task. However, when these same individuals were instructed not to follow commands, none returned a positive outcome. Evidently, any automatic brain responses generated by participants listening to the instructions are not sufficient for significant task performance; rather, an act of consistently timed, volitional command following is needed. Furthermore, in all three of the 16 patients who showed significant EEG outcomes after commands, EEG activity before the commands was not classifiable, which provides further evidence that they were all producing task-appropriate EEG responses in time with the cues, as required by the task instructions. Therefore, successful completion of these EEG tasks represents a substantial cognitive feat, not only for patients who were presumed to be vegetative, but also for control participants.

To be deemed successful, each respondent must have consistently generated the requested mental states to command for a prolonged period within each trial, and must have done so for several trials. One patient produced consistently appropriate EEG responses for about 100 trials (table). 11 of the 12 controls produced EEG data that were less accurately classified than this patient. Conversely, all patients were tested with the CRS-R for at least 4 days, and at no point did any patient show a behavioural sign of awareness (eg, visual fixation, visual pursuit, localisation to pain). More importantly, none showed any evidence of a residual ability to respond to command. Therefore, these patients were not misdiagnosed in the normal sense of

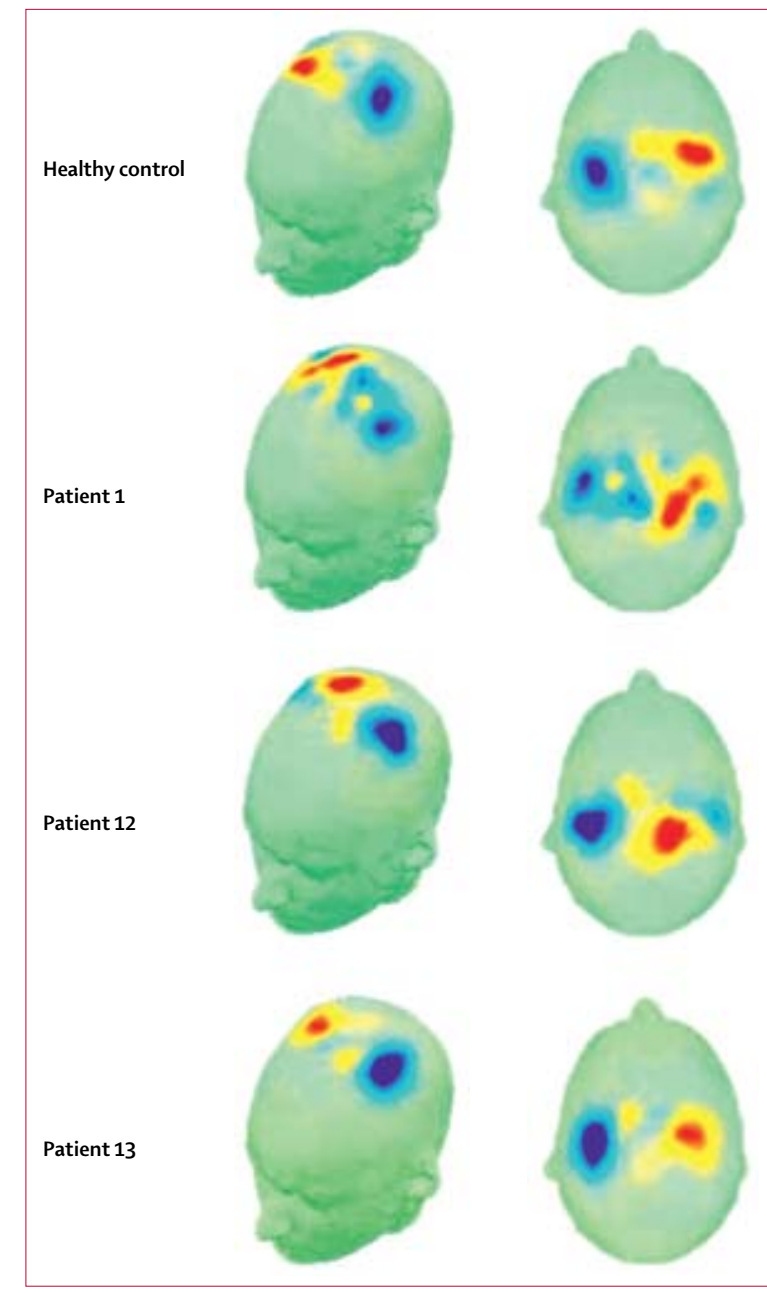

Figure 2: Modulation of the appropriate frequency bands of the EEG signal that are associated with motor imagery in three patients and one healthy control participant

Maps show the scalp distribution of the feature (timepoint $x$ frequency band) with the highest absolute coefficient value from one training run of the cross-validation procedure. Red colours show coefficient values greater than zero. Blue colours show values less than zero. When the scalp distributions of data for the classification procedure are plotted, the neurophysiological basis of the positive EEG outcomewith clear foci over the hand and toe motor areas-are formally identical when compared between a healthy control participant and the three patients in the vegetative state who significantly followed commands with this EEG task.

the word. Rigorous assessments by experienced teams showed they were all correctly diagnosed according to existing behavioural criteria. Clearly however, those criteria did not adequately identify the actual condition of these patients in at least $19 \%$ of cases.

What then, is the appropriate diagnosis for these patients who can follow command with an EEG response, but with no overt physical behaviour? We cannot draw strong conclusions about the inner worlds of these patients based solely on an ability to generate accurate and consistent EEG responses to command. However, performance of this complex task does make several demands on many cognitive functions, including sustained attention (more 
Panel: Research in context

\section{Systematic review}

We searched PubMed and Web of Science from July, 2010, to June, 2011, with the terms "vegetative state" and "awareness". We assessed the evidence on the basis of quality of the scientific methods and used only publications written in English.

\section{Interpretation}

The prevalence of covert command following in our cohort of patients diagnosed as being in the vegetative state accords with studies of functional MRI, and reinforces the evidence that a notable portion of this patient group retain awareness that is not consistent with their externally observable behaviour. Our findings show that covert command following could be detected at the bedside of a vegetative patient with use of EEG-a cheaper and more accessible method than current techniques of assessment. Therefore, this method could reach all vegetative patients and fundamentally change their bedside assessment.

than 90-s blocks), response selection (between the two imagery tasks), language comprehension (of the task instructions), and working memory (to remember which task to do for many trials within each block). All these functions are aspects of top-down cognitive control, which are usually associated with, and could characterise, normal conscious awareness. ${ }^{15}$ Future studies should further characterise the residual cognitive abilities in this subset of patients and how they contribute to commandfollowing. However, our findings of succesful commandfollowing in patients characterised as vegetative state show that functional neuroimaging-and EEG specifically-is better suited for providing such a characterisation than are existing methods of clinical assessment.

Several reasons could explain the range of significant classification accuracies for patients and controls. First, brain-state classification with no previous training for the individual has produced low classification accuracies in healthy participants (eg, about 75\% for right-hand vs feet imagery $^{8}$ ), and the same would be true for any patient group. Second, differences in attention or capabilities of the working memory could have had a role in the variance of classification accuracies within the patient group. Indeed, a patient whose diminished working memory leads them to forget the instructions for a particular block after ten tones for example, will produce EEG noise for the classifier only in the remaining five tones, leading to reduced classification accuracy.

Why could three controls in our study not produce significant EEG records? Participants who receive no feedback or training in imagery tasks are likely to produce relatively lower classification accuracies compared with trained individuals. Furthermore, some healthy individuals might be unable to produce reliable classification, even with feedback training ${ }^{10}$ (so-called brain-computer interface illiterates). The absence of a positive EEG outcome for three (aware) controls emphasises the importance of the interpretation of only positive results in patients, because this finding shows unequivocally that a null EEG outcome does not necessarily indicate an absence of awareness. Alongside behavioural assessment and other functional neuroimaging approaches, ${ }^{16}$ many testing sessions for several days with this EEG technique will provide each patient with an increased opportunity to show their covert awareness, if it exists.

The method described here could fundamentally change the assessment of this complex patient group because EEG is highly portable, inexpensive, can be done at the bedside, is available in most hospitals, and can be used with patients who have metal implants. Moreover, in the most comprehensive study of fMRI so far, data for nine (17\%) of 54 patients could not be interpreted because of excessively noisy data from motion artifacts. ${ }^{5}$ By comparison, EEG is less affected by small motion artifacts, resulting in a drop-out rate of zero in our study. Our findings show that consistent responses to command need not be expressed behaviourally, but can be identified accurately on the basis of EEG responses. The success of this technique also paves the way for development of brain-computer interfaces ${ }^{17}$-or simple, reliable communication devices-in this patient group. Such devices will provide a form of external control and communication based on mappings of distinct mental states (eg, patients imagining right-hand movements to communicate yes, and toe movements to communicate no). The degrees of freedom provided by EEG could take this technique beyond binary responses to allow methods of communication that are far more functionally expressive, based on many forms of mental state classification. ${ }^{18-20}$ The development of techniques for the real-time classification of these forms of mental imagery will enable routine two-way communication with some of these patients, allowing them to share information about their inner worlds, experiences, and needs.

\section{Contributors}

DC designed the EEG task; obtained and analysed data for healthy controls and for patients from Cambridge, UK; developed the analyses methods; and wrote the paper. SC developed the analyses methods and analysed all patient data. CC did the behavioural and EEG assessments of all patients at University Hospital of Liège, Belgium. TAB and DF-E provided conceptual input. JDP was the clinician responsible for patients at Addenbrooke's Hospital, Cambridge, UK. SL was the clinician responsible for all patients at University Hospital of Liège, Belgium. AMO provided conceptual advice and wrote the manuscript. All other authors contributed to the final version.

Conflicts of interest

We declare that we have no conflicts of interest.

\section{Acknowledgments}

This study was funded by the Medical Research Council, the James S McDonnell Foundation, the Canada Excellence Research Chairs Program, the European Commission, Fonds de la Recherche Scientifique, the Mind Science Foundation, the Belgian French-Speaking Community Concerted Research Action, University Hospital of Liège, and the University of Liège. We thank Judith Allinson and Evelyn Kamau (Department of Clinical Neurosciences, Cambridge University), and 
Beth Parkin (Medical Research Council Cognition and Brain Sciences Unit, Cambridge) for admission and assessment of patients at Addenbrooke's Hospital, Cambridge; and Guy Williams and Tom Ash (Deptartment of Clinical Neurosciences, Cambridge University), and Fraser Smith (Centre for Brain and Mind, University of Western Ontario) for their methodological input.

\section{References}

1 Schnakers C, Vanhaudenhuyse A, Giacino J, et al. Diagnostic accuracy of the vegetative and minimally conscious state: clinical consensus versus standardized neurobehavioral assessment. BMC Neurol 2009; 9: 35.

2 Childs NL, Mercer WN, Childs HW. Accuracy of diagnosis of persistent vegetative state. Neurology 1993; 43: 1465-67.

3 Andrews K, Murphy L, Munday R, Littlewood C. Misdiagnosis of the vegetative state: retrospective study in a rehabilitation unit. BMJ 1996; 313: 13-16.

4 Owen AM, Coleman MR, Boly M, Davis MH, Laureys S, Pickard JD. Detecting awareness in the vegetative state. Science 2006; 313: 1402.

5 Monti MM, Vanhaudenhuyse A, Coleman MR, et al. Willfu modulation of brain activity in disorders of consciousness. N Engl J Med 2010; 362: 579-89.

6 Vaughan TM, McFarland DJ, Schalk G, et al. The Wadsworth BCI Research and Development Program: at home with BCI. IEEE Trans Neural Syst Rehabil Eng 2006; 14: 229-33.

7 Pfurtscheller G, Neuper C. Motor imagery activates primary sensorimotor area in humans. Neurosci Lett 1997; 239: 65-68.

8 Pfurtscheller G, Scherer R, Muller-Putz GR, Lopes da Silva FH. Short-lived brain state after cued motor imagery in naive subjects. Eur J Neurosci 2008; 28: 1419-26.

9 Pfurtscheller G, Brunner C, Schl` ${ }^{\wedge}$ gl A, Lopes da Silva FH.

Mu rhythm (de)synchronization and EEG single-trial classification of different motor imagery tasks. NeuroImage 2006; 31: 153-59.
10 Guger C, Edlinger G, Harkam W, Niedermayer I, Pfurtscheller G. How many people are able to operate an EEG-based brain-computer interface (BCI)? IEEE Trans Neural Syst Rehabil Eng 2003; 11: 145-47.

11 Kalmar K, Giacino JT. The JFK Coma Recovery Scale-Revised. Neuropsychol Rehabil 2005; 15: 454-60.

12 Delorme A, Makeig S. EEGLAB: an open source toolbox for analysis of single-trial EEG dynamics including independent component analysis. J Neurosci Methods 2004; 134: 9-21.

13 Pfurtscheller G, Neuper C, Berger J. Source localization using eventrelated desynchronization (ERD) within the alpha band. Brain Topogr 1994; 6: 269-75.

14 Scholkopf B, Smola AJ. Learning with Kernels. Cambridge, MA: MIT Press, 2002.

15 Naccache L. Psychology. Is she conscious? Science 2006; 313: 1395-6.

16 Bekinschtein TA, Dehaene S, Rohaut B, Tadel F, Cohen L Naccache L. Neural signature of the conscious processing of auditory regularities. Proc Natl Acad Sci USA 2009; 106: 1672-77.

17 Birbaumer N. Breaking the silence: Brain-computer interfaces (BCI) for communication and motor control. Psychophysiology 2006; 43: 517-32.

18 Farwell LA, Donchin E. Talking off the top of your head: toward a mental prosthesis utilizing event-related brain potentials. Electroencephalogr Clin Neurophysiol 1988; 70: 510-23.

19 Sellers EW, Donchin E. A P300-based brain-computer interface: initial tests by ALS patients. Clin Neurophysiol 2006; 117: 538-48.

20 Wolpaw JR, Birbaumer N, McFarland DJ, Pfurtscheller G, Vaughan TM. Brain-computer interfaces for communication and control. Clin Neurophysiol 2002; 113: 767-91. 


\section{Measurements of consciousness in the vegetative state}

In The Lancet, Damian Cruse and colleagues' study ${ }^{1}$ examines 16 patients in the vegetative state. This disorder is defined as wakefulness without conscious awareness of self and environment. The investigators showed that three (19\%) of the 16 patients could generate EEG responses to two commands involving motor imagery, although the patients were otherwise behaviourally unresponsive. But how can this discovery be understood?

Researchers typically accept a distinction between the contents and levels of consciousness. ${ }^{2}$ Contents of consciousness are defined as subjective experienceeg, the taste of coffee, feeling of pain, or experience of the colour red. In the study of levels of consciousness, ${ }^{2}$ three distinct stages of degraded consciousness have been described: coma, the vegetative state, and the minimally conscious state. Differentiation between the stages is based on behavioural criteria. Patients in the vegetative state differ from those in a coma because they can be aroused, yet both groups are considered fully unconscious. Patients in the minimally conscious state are believed to have fluctuating consciousness and are distinguished from the vegetative state when an outside observer (a doctor in most cases) thinks the patient has a minimum understanding of self or the environment (eg, a voluntary attempt to communicate). Other patients with severe brain injury, who are not in the minimally conscious state, are typically believed to be more conscious ${ }^{3}$ than minimally conscious patients.

Immediate problems occur with attempts to operationalise these definitions, not least with the aim to develop a bedside test for consciousness. Levels of consciousness, in the standard scale, are defined as perceptible signs of being in contact with the outside world. Whereas this definition might make intuitive sense, it is not identical to subjective experience. In cognitive science, nearly all mental events exist with and without consciousness. Complex types of behaviour, response inhibition, task switching, instruction following, conflict monitoring, and error detection have all been described as fully functional in the absence of reportable conscious experience. ${ }^{4}$ Similarly, complex aspects of perception, such as semantic interpretation, can occur unconsciously. ${ }^{5}$

No patient has been discovered who could follow instructions, yet was completely unconscious. However, with the scarcity of external validation methods in the study of consciousness, to imagine how such a patient could be identified is difficult. Yet findings from some studies $^{6}$ indicate that so-called split-brain patients react to commands without (reported) awareness of the command. From a different perspective, many published works ${ }^{7}$ discuss the possible dissociation between the neural substrate of vision for action and vision for perception (and perceptual consciousness). Therefore, command following, which was used as an objective indicator for consciousness in Cruse and colleagues' study, ${ }^{1}$ might not be an absolute measure for identification of whether a person is conscious. Because three $(25 \%)$ of the 12 healthy controls in this study could not produce significant EEG records, command following most likely measures something different than the presence and absence of consciousness.

Nevertheless, the new discovery by Cruse and colleagues is surprising and challenging. The study continues a line of research with other revolutionary findings-eg, that of Owen and colleagues, ${ }^{8}$ who instructed a patient in the vegetative state to "imagine playing tennis" or "imagine visiting the rooms in your house". The resulting brain activation was no different from that of control participants. In a follow-up study, ${ }^{9}$ this technique was applied to communicate successfully with five of 54 patients in the vegetative state, by asking them to think about tennis for "yes", and being in their house for "no". With Cruse and colleagues' study, ${ }^{1}$ these

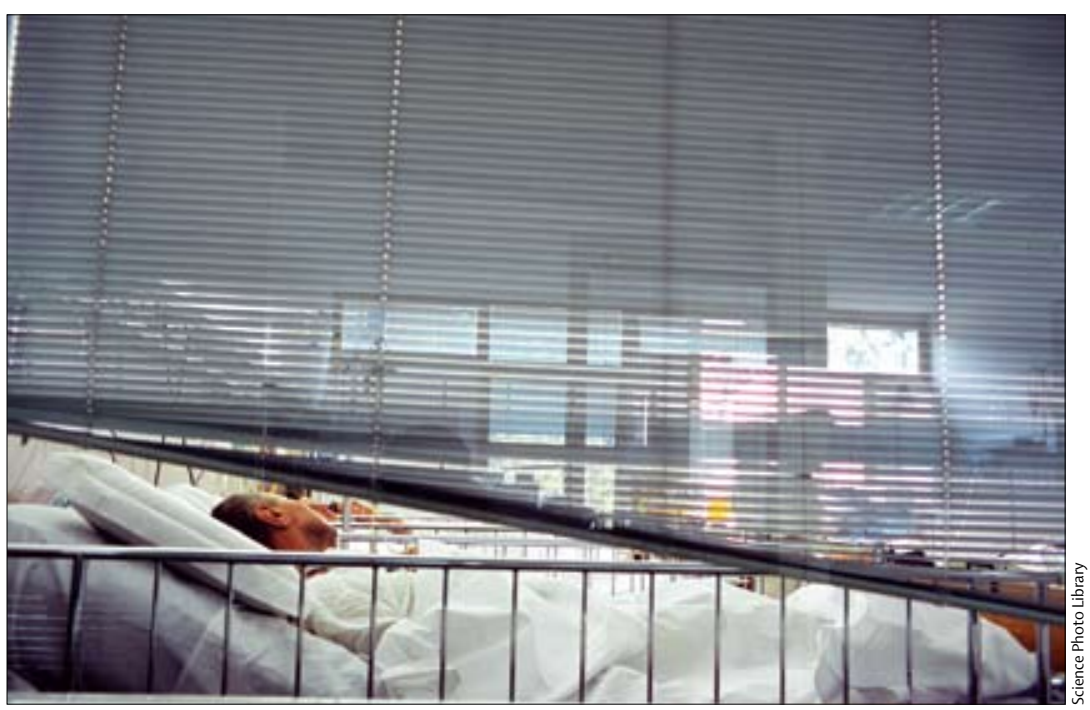

Published Online November 10, 2011 DOI:10.1016/S01406736(11)61591-2 See Online/Articles DOI:10.1016/S01406736(11)61224-5 
findings present good evidence that at least some patients in the vegetative state are conscious. However, the methods in all these experiments are indirect and investigate a factor other than consciousness alone.

So far, most researchers have interpreted these published results as suggesting that many patients in the vegetative state are wrongly diagnosed; however, these studies have an even stronger consequence. The real underlying issue is that the levels of consciousness have little to do with consciousness-ie, subjective experience. A more plausible interpretation is that vegetative and minimally conscious states distinguish between different levels of cognitive and communicative abilities, which is a different matter than subjective experience per se. A new classification system is necessary if the goal is to understand the cognitive functioning of patients in the vegetative or minimally conscious states. Such a system should begin with a much more explicit attempt to use objective methods that have been correlated with reports of subjective experience in healthy individuals. ${ }^{10}$

\section{* Morten Overgaard, Rikke Overgaard}

Cognitive Neuroscience Research Unit (CNRU), Department of Communication and Psychology, Aalborg University,

9220 Aalborg Oest, Denmark (MO); and Hammel

Neurorehabilitation and Research Center (MO), and CNRU Centre of Functionally Integrative Neuroscience (RO), MindLab, Aarhus University, Aarhus, Denmark

mortover@rm.dk
MO was funded by a Starting Grant from the European Research Council. We declare that we have no conflicts of interest.

1 Cruse D, Chennu S, Chatelle $C$, et al. Bedside detection of awareness in the vegetative state: an observational cohort study. Lancet 2011; published online Nov 10. DOI:10.1016/S0140-6736(11)61224-5.

2 Laureys $\mathrm{S}$. The neural correlate of (un)awareness: lessons from the vegetative state. Trends Cogn Sci 2005; 9: 556-59.

3 Overgaard M. How can we know if patients in coma, vegetative state or minimal conscious state are conscious? Prog Brain Res 2009; 177: 11-19.

4 van Gaal S, Lamme VA. Unconscious high-level processing: Implications for neurobiological theories of consciousness. Neuroscientist 2011; published online May 31. DOI:10.1177/1073858411404079.

5 Marcel AJ. Conscious and unconscious perception: an approach to the relations between phenomenal experience and perceptual process. Cogn Psychol 1983; 15: 238-300.

6 Cooney JW, Gazzaniga MS. Neurological disorders and the structure of human consciousness. Trends Cogn Sci 2003; 7: 161-65.

7 Milner AD, Goodale MA. Two visual systems re-viewed. Neuropsychologia 2008; 46: 774-75.

8 Owen AM, Coleman MR, Boly M, Davis MH, Laureys S, Pickard J. Detecting awareness in the vegetative state. Science 2006; 313: 1402.

9 Monti MM, Vanhaudenhuyse A, Coleman MR, et al. Willful modulation of brain activity in disorders of consciousness. N Engl J Med 2010; 362: 579-89.

10 Overgaard M, Overgaard R. Neural correlates of contents and levels of consciousness. Front Psychol 2010; 1: 1-3. 